\title{
Heterogeneous Multifrequency Direct Inversion (HMDI) for magnetic resonance elastography with application to a clinical brain exam
}

\author{
Eric Barnhill ${ }^{\mathrm{a}, *}$, Penny J. Davies ${ }^{\mathrm{b}}$, Cemre Ariyurek ${ }^{\mathrm{c}}$, Andreas Fehlner ${ }^{\mathrm{d}}$, Jürgen Braun ${ }^{\mathrm{d}}$, \\ Ingolf Sack ${ }^{\mathrm{a}}$
}

a Department of Radiology, Charité Universitätsmedizin Berlin, Berlin, Germany

${ }^{\mathrm{b}}$ Department of Mathematics and Statistics, University of Strathclyde, Glasgow, Scotland, United Kingdom

${ }^{c}$ Department of Electrical and Electronics Engineering, Bilkent University, Ankara, Turkey

${ }^{\mathrm{d}}$ Institute for Medical Informatics, Charité Universitätsmedizin Berlin, Berlin, Germany

\section{A R T I C L E I N F O}

\section{Article history:}

Received 5 May 2017

Revised 9 March 2018

Accepted 13 March 2018

Available online 17 March 2018

\section{Keywords:}

Elastography

Magnetic resonance imaging

Magnetic resonance elastography

Viscoelasticity

Inverse problems

\begin{abstract}
A B S T R A C T
A new viscoelastic wave inversion method for MRE, called Heterogeneous Multifrequency Direct Inversion (HMDI), was developed which accommodates heterogeneous elasticity within a direct inversion (DI) by incorporating first-order gradients and combining results from a narrow band of multiple frequencies. The method is compared with a Helmholtz-type DI, Multifrequency Dual Elasto-Visco inversion (MDEV), both on ground-truth Finite Element Method simulations at varied noise levels and a prospective in vivo brain cohort of 48 subjects ages 18-65. In simulated data, MDEV recovered background material within $5 \%$ and HMDI within $1 \%$ of prescribed up to SNR of $20 \mathrm{~dB}$. In vivo HMDI and MDEV were then combined with segmentation from SPM to create a fully automated "brain palpation" exam for both whole brain (WB), and brain white matter (WM), measuring two parameters, the complex modulus magnitude $\left|G^{*}\right|$, which measures tissue "stiffness", and the slope of $\left|G^{*}\right|$ values across frequencies, a measure of viscous dispersion. $\left|G^{*}\right|$ values for MDEV and HMDI were comparable to the literature (for a 3-frequency set centered at $50 \mathrm{~Hz}$, WB means were 2.17 and $2.15 \mathrm{kPa}$ respectively, and WM means were 2.47 and $2.49 \mathrm{kPa}$ respectively). Both methods showed moderate correlation to age in both WB and WM, for both $\left|G^{*}\right|$ and $\left|G^{*}\right|$ slope, with Pearson's $r \geq 0.4$ in the most sensitive frequency sets. In comparison to MDEV, HMDI showed better preservation of recovered target shapes, more noise-robustness, and stabler recovery values in regions with rapid property change, however summary statistics for both methods were quite similar. By eliminating homogeneity assumptions within a fast, fully automatic, regularization-free direct inversion, HMDI appears to be a worthwhile addition to the MRE image reconstruction repertoire. In addition to supporting the literature showing decrease in brain viscoelasticity with age, our work supports a wide range of inter-individual variation in brain MRE results.
\end{abstract}

(c) 2018 Elsevier B.V. All rights reserved.

\section{Introduction}

Magnetic resonance elastography (MRE) (Hirsch et al., 2016) encodes induced shear wave displacements using phase-contrast MRI imaging, enabling estimation of in vivo tissue viscoelastic properties by wave inversion. These mechanical properties, including mechanical "stiffness" and viscosity, are of strong medical interest (Mariappan et al., 2010; Sack et al., 2013), and elastographyrelated research is widespread. One of the strengths of MRE is

\footnotetext{
* Corresponding author.

E-mail addresses: eric.barnhill@charite.de, ericbarnhill@protonmail.ch (E. Barnhill), penny.davies@strath.ac.uk (P.J. Davies), cemre@ee.bilkent.edu.tr (C. Ariyurek), andreas.fehlner@charite.de (A. Fehlner), juergen.braun@charite.de (J. Braun), ingolf.sack@charite.de (I. Sack).
}

that full-field displacements of tissue are acquired; however magnetic resonance elastography is challenged by complicated tissue structures and the ill-posed nature of wave inversion. The needed regularization techniques can reduce effective resolution elements well below that of the MRI acquisition voxel size, and some solutions require manual intervention. There is thus an ongoing interest in magnetic resonance elastography elasticity reconstruction techniques that relax regularization constraints within a robust and fully automated image processing pipeline.

In the present study a new approach to magnetic resonance elastography stiffness reconstruction is described, called heterogeneous multifrequency direct inversion, a direct inversion method which admits heterogeneity while leaving boundaries free. Such an approach potentially advances direct inversion by accommodating heterogeneity in stiffness within a fully automated pipeline. The 
method is validated through the use of Finite Element Method simulations and compared with the more common form of direct inversion, which neglects stiffness gradients, known as Algebraic Helmholtz Inversion (Papazoglou et al., 2008). As both direct inversion methods are fast and automatic, we investigate their performance in a fully automated "brain palpation" exam which combines direct inversion with image segmentation and analysis algorithms from SPM (Friston et al., 1995) to measure stiffness and viscosity of whole brain and white matter across the lifespan.

\subsection{Background}

Magnetic resonance elastography wave inversion usually applies the Navier-Lamé equation for conservation of linear momentum in isotropic viscoelastic solids

$\left(\mu\left(u_{i, j}+u_{j, i}\right)\right)_{, j}+(\lambda \operatorname{div} \mathbf{u})_{, i}=\rho \ddot{u}_{i}$

Where $\mathbf{u}$ is the $3 \mathrm{D}$, vector-valued time-harmonic displacement field of the material, $\lambda$ and $\mu$ are the first and second Lamé parameters, $\rho$ is the density, div is the divergence operator, and body forces are assumed zero. Typically, magnetic resonance elastography applies steady-state vibration at driving frequency $\omega$, assumes a uniform density set to that of water, and removes the divergence of the displacement field, reducing Eq. (1) to

$\mu \nabla \cdot\left(\nabla \mathbf{u}+\nabla^{T} \mathbf{u}\right)+\nabla \mu \cdot\left(\nabla \mathbf{u}+\nabla^{T} \mathbf{u}\right)=-\rho \omega^{2} \mathbf{u}$

where the shear term of Eq. (1) has been expanded using a vector calculus product rule. As Eq. (2) contains 4 unknowns (the shear modulus $\mu$ and its three directional gradients $\nabla \mu$ ) but only 3 scalar displacement fields (the $\mathrm{x}, \mathrm{y}$, and $\mathrm{z}$ displacement components of $\mathbf{u}$ ), a boundary condition needs to be imposed on $\mu$ in order to be well-posed and thus produce a unique solution for $\mu$ (Sánchez et al., 2010).

In the magnetic resonance elastography literature this difficulty been handled one of three ways. The simplest model is to neglect $\nabla \mu$, or assume "local homogeneity". Such an approach reduces the Navier-Lamé equation to an algebraic form

$\mu=\rho \omega^{2} \mathbf{u} / \nabla^{2} \mathbf{u}$

where $\nabla^{2}$ is the Laplacian operator. This approach implicitly assumes an infinite boundary condition, and would only be accurate to the extent the gradient of $\mu$ is really neglectable. As $\mathbf{u}$ is complex-valued, Eq. (3) yields a complex shear modulus with both storage and loss information, and this technique has been reported as Algebraic Inversion of the Differential Equation (AIDE) (Oliphant et al., 2001; Manduca et al., 2001) and later as Algebraic Helmholtz Inversion (AHI) (Papazoglou et al., 2008).While the imaginary component of $\mu$ holds diagnostic potential, some further reformulation can increase robustness for the real component, or the magnitude, of this modulus, which is the value commonly measured. Local Frequency Estimation (LFE) neglects attenuation and so estimates a real "shear stiffness" by combining local wavenumber estimates at multiple scales (Manduca et al., 2001) which is more robust to noise. The Multifrequency Dual Elasto-Visco Inversion method (Papazoglou et al., 2012) delivers a complex modulus magnitude by handling only the magnitudes of the displacement and Laplacian field images. This better handles violations of the model assumptions such as boundaries, as the values remain positive and tend to zero. (Multifrequency Dual Elasto-Visco Inversion also fuses multifrequency results and this is discussed below.) Nonetheless limitations to this approach include artifact at discontinuities, inaccuracies at small features or within regions of rapid change, and an increase in noise from the enforcement of local homogeneity prior to inversion.
More complex inversion models that incorporate heterogeneity of $\mu$ are gaining in use. A well-established technique is to integrate Eq. (2) using displacement field values at the boundaries, reducing modulus recovery to a Dirichlet-type problem (Van Houten et al., 1999). Limitations to this approach include the need for masking to set and determine boundaries, either manually or by algorithm; overweighting of the values used for boundaries relative to the other displacements (Park and Maniatty, 2006), and the reported need for smoothing, and thus reduction in image resolution, to achieve stability (Park and Maniatty, 2006; McGarry, 2013).

In recent mathematical work, Sánchez et al. (2010) showed that a unique, direct inversion-based solution for $\mu$ can be obtained by overdetermining the results from two or more linearly decorrelated displacements and leaving boundaries free. This proposed approach has several potential advantages: it is computationally inexpensive; no masking or marking is required; no regularization is required for stability; and heterogeneities in stiffness are accommodated. A straightforward approach to obtaining such decorrelated acquisitions is to acquire multiple data sets at varied driving frequencies within a narrow band, and assume that the known wide-band frequency dispersion of the shear modulus (Szabo, 1995) can be neglected. Such a "multifrequency" approach has been previously applied to magnetic resonance elastography , to better condition both the heterogeneous (Honarvar et al., 2013) and homogeneous (Papazoglou et al., 2012) forms of the inversion problem.

\subsection{Aims of the paper}

In the present study, we apply the uniqueness findings of Sánchez et al. (2010) to serial multifrequency acquisitions, in combination with sparsity-promoting image processing, to directly overdetermine $\mu$ without neglecting gradients, estimating boundaries, or smoothing, a pipeline here called heterogeneous multifrequency direct inversion. The study aims to:

1. Deliver the first heterogeneity accommodating direct inversion images of in vivo magnetic resonance elastography data

2. Validate the method against ground-truth Finite Element Method simulations with a range of noise (and hence signalto-noise ratio) values

3. Evaluate the performance of the method against Helmholtz inversion in the Finite Element Method and in vivo cases

4. Exploiting that both methods require no manual intervention, evaluate their performance in a fully automated "brain palpation" exam which incorporates automated segmentation and co-registration from SPM.

A prospective data set spanning the adult human lifespan was acquired for the study, allowing us to compare not only stiffness values with the rest of the literature, but investigate whether we detect the previously reported relationship between age and viscoelasticity decrease (Arani et al., 2015; Sack et al., 2009) as well as whether aging effects interact with frequency.

\section{Methods}

\subsection{Heterogeneous multifrequency direct inversion}

In heterogeneous multifrequency direct inversion, the divergence-free displacement fields $\mathbf{u}_{1 . n}$ and their derivatives are "stacked" within a single block matrix system to overdetermine $\mu$ : 


$$
\begin{aligned}
& {\left[\begin{array}{cc}
\nabla \cdot\left(\nabla \mathbf{u}+\nabla^{T} \mathbf{u}\right)_{1} & \left(\nabla \mathbf{u}+\nabla^{T} \mathbf{u}\right)_{1} \\
\nabla \cdot\left(\nabla \mathbf{u}+\nabla^{T} \mathbf{u}\right)_{2} & \left(\nabla \mathbf{u}+\nabla^{T} \mathbf{u}\right)_{2} \\
\ldots & \\
\nabla \cdot\left(\nabla \mathbf{u}+\nabla^{T} \mathbf{u}\right)_{n} & \left(\nabla \mathbf{u}+\nabla^{T} \mathbf{u}\right)_{n}
\end{array}\right]\left[\begin{array}{c}
I \\
\nabla^{T}
\end{array}\right] \mu} \\
& =-\rho\left[\begin{array}{llll}
\omega_{1} & & \\
& \omega_{2} & & \\
& & \ldots & \\
& & \omega_{n}
\end{array}\right]\left[\begin{array}{c}
\mathbf{u}_{1} \\
\mathbf{u}_{2} \\
\ldots \\
\mathbf{u}_{n}
\end{array}\right]
\end{aligned}
$$

where, in addition to terms identified earlier, $n$ is the frequency index and $I$ is the identity matrix.

This equation can be compared with Eq. (3) in (Sánchez et al., 2010), however in that paper, it is proposed to either use decorrelated fields at the same frequency, or use single fields in which special conditions obtain that ensure uniqueness (likely obtained in real-world data by projecting the data onto a subspace with the desired properties). As acquisitions at different frequencies are guaranteed to be orthogonal, by extending this method to multiple frequencies we resolve these uniqueness and conditioning concerns without further filtering of the data, which would be likely to have a smoothing effect.

Multiplying through the first two matrices on the left hand side to obtain

$$
\mathbf{A}=\left[\begin{array}{cc}
\nabla \cdot\left(\nabla \mathbf{u}+\nabla^{T} \mathbf{u}\right)_{1} & \left(\nabla \mathbf{u}+\nabla^{T} \mathbf{u}\right)_{1} \\
\nabla \cdot\left(\nabla \mathbf{u}+\nabla^{T} \mathbf{u}\right)_{2} & \left(\nabla \mathbf{u}+\nabla^{T} \mathbf{u}\right)_{2} \\
\ldots & \\
\nabla \cdot\left(\nabla \mathbf{u}+\nabla^{T} \mathbf{u}\right)_{n} & \left(\nabla \mathbf{u}+\nabla^{T} \mathbf{u}\right)_{n}
\end{array}\right]\left[\begin{array}{c}
I \\
\nabla^{T}
\end{array}\right]
$$

and multiplying through the terms on the right-hand side to obtain

$b=-\rho\left[\begin{array}{cccc}\omega_{1} & & & \\ & \omega_{2} & & \\ & & \ldots & \\ & & & \omega_{n}\end{array}\right]^{2}\left[\begin{array}{c}\mathbf{u}_{1} \\ \mathbf{u}_{2} \\ \ldots \\ \mathbf{u}_{n}\end{array}\right]$

reduces the stacked matrix to the familiar form $A \mu=b$ wherein it is easily seen that $\mu$ can be obtained by $\mu=A^{-1} b$. GNU Octave (Eaton et al., 2015) and Matlab (Mathworks, Natick, MA) compatible code for the method is provided in Supplementary Information.

\section{2. $M D E V$}

Neglect of the first-order gradients in Eq. (4) leads to an overdetermined least-squares inversion across $\omega_{1} \ldots \omega_{n}$ for $n \geq 1$, however, the conditioning of the problem can be improved with three reformulations.

First, the local homogeneity assumption reduces the shear term in Eq. (1) to $\mu u_{i, j j}$. This enables replacement of the tensor divergence $\nabla \cdot \epsilon$ with the vector Laplacian $\nabla^{2} \mathbf{u}$. Second, shear modulus magnitude, usually notated as $\left|G^{*}\right|\left(G^{*} \equiv \mu\right)$, can be recovered treating only the magnitude quantities, $|\mathbf{u}|$ and $\left|\nabla^{2} \mathbf{u}\right|$, which do not show the same outlier behavior as the corresponding complex quantities at discontinuities and other violations of local homogeneity - they remain positive and tend toward zero. Third, the least-squares solution projects $\mathbf{u}$ onto the space of the derivatives (shown in Oliphant et al., 2001), which are more sensitive to noise; instead $|\mathbf{u}|$ and $\left|\nabla^{2} \mathbf{u}\right|$ can be averaged, that is projected onto the ones vector (Braun et al., 2014), as the averaged value is also the barycentre. These observations lead to the Multifrequency Dual
Elasto-Visco Inversion inversion equation:

$$
\left|G^{*}\right|=\rho \frac{\sum_{m=1}^{3} \sum_{n=1}^{N} \omega_{n}^{2}\left|u_{m}\left(\omega_{n}\right)\right|}{\sum_{m=1}^{3} \sum_{n=1}^{N}\left|\nabla^{2} u_{m}\left(\omega_{n}\right)\right|}
$$

Where $m$ is the directional component index, $n$ the frequency index, and $u$ the scalar displacement field. Applied in many brain studies (e.g. Guo et al., 2013; Fehlner et al., 2017; Streitberger et al., 2014), Multifrequency Dual Elasto-Visco Inversion is here compared with heterogeneous multifrequency direct inversion.

\subsection{Image processing pipeline}

Identical pre- and post-processing was used for Multifrequency Dual Elasto-Visco Inversion and heterogeneous multifrequency direct inversion data:

Phase unwrapping The data were phase-unwrapped using PhaseTools' Laplacian Based Estimate (Barnhill et al., 2014)

Denoising The complex wavefields were denoised in a complex dual-tree wavelet basis (Barnhill et al., 2017; Selesnick et al., 2005) with soft thresholding and VisuShrink (Donoho and Johnstone, 1995) threshold estimation. Here an 3D undecimated discrete wavelet transform was used in place of the critically sampled transform used in Barnhill et al. (2017) to eliminate computational demands from cycle spinning. Median absolute deviation estimates (Gauss, 1816) for VisuShrink were masked to anatomical regions of the complex wave volume (obtained by thresholding the T2 magnitude image). The VisuShrink estimate was vectorial, incorporating all three dimensions of wave propagation simultaneously.

Divergence removal As bulk wave wavelengths are estimated to be over an order of magnitude larger than shear (Manduca et al., 2001; Sinkus et al., 2005), very low frequencies were removed from the image using a $3 \mathrm{D}, 4$ th order Butterworth high pass filter with normalized frequency cutoff of 0.05 .

Segmentation (brain only) Post-inversion, the averaged $\mathrm{T2}^{*}$ weighted magnitude image from the multifrequency acquisition was transformed and segmented in MNI space (Evans et al., 2012) with statistical parametric mapping (Friston et al., 1995) and the deformation and segmentation matrices were applied to the $\left|G^{*}\right|$ maps to obtain regional measurements, similar to e.g. Guo et al. (2013) and Fehlner et al. (2017). As the slab was incomplete, thresholding was required to accurately evaluate partially acquired regions; labeled regions were thresholded using a minimum cut-off of $500 \mathrm{~Pa}$, which was more than two standard deviations above measured elastogram noise but below all measured anatomical values.

In this study derivatives were estimated by centered differences after denoising. This procedure varies from some previously published approaches using polynomial fit derivative estimates (e.g. Oliphant et al., 2001) or derivative estimates from polynomial shape functions (e.g. Honarvar et al., 2013). Such polynomial estimates are not noise-adaptive, which may be a source of error as their accuracy to an underlying interpolated polynomial is a function of noise level (Knowles and Renka, 2014). Such fits will further be a function of the window over which they are estimated. Here noise is removed using wavelets, which will adapt to noise while preserving boundaries, with the ensuing finite difference estimates measuring the derivative in the smallest possible stable region. 


\subsection{Finite element simulation}

For ground truth evaluations a Finite Element Method simulation was generated using ABAQUS (Dassault Systèmes, France) using the methodology published in Hollis et al. (2016). Voigt-model material was chosen and the mesh elements were isotropic hexahedrons of $1 \mathrm{~mm} \times 1 \mathrm{~mm} \times 1 \mathrm{~mm}$ cubic size, with overall simulation size $80 \mathrm{~mm} \times 100 \mathrm{~mm} \times 10 \mathrm{~mm}$. The simulation consisted of four cylindrical targets of $9 \mathrm{kPa}$ stiffness, of radii $20 \mathrm{~mm}$, $10 \mathrm{~mm}, 4 \mathrm{~mm}$ and $2 \mathrm{~mm}$ respectively, within a background material of $3 \mathrm{kPa}$, and both materials with shear viscosity of $1 \mathrm{~Pa} \cdot \mathrm{s}$. The simulation material was subjected to simulated steady state shear wave vibration at $50-90 \mathrm{~Hz}$ in steps of $10 \mathrm{~Hz}$ from a surface traction on the top $x z$ plane, and the other boundaries of the box were absorbent. Phase field noise can be estimated as Gaussian for SNR > $2 \mathrm{~dB}$ (Gudbjartsson and Patz, 1995) so the robustness of the method to noise was tested using a range of Gaussian noise levels as previously done in Barnhill et al. (2017). The Finite Element Method data were compared by:

Recovery method The data were recovered using heterogeneous multifrequency direct inversion and multifrequency Dual Elasto-Visco Inversion

Number of frequencies The data were recovered combining all 5 frequencies, as well as sliding widows of 3 frequencies each, in accordance with the in vivo experimental design below

Noise levels Gaussian noise levels from 50 to $10 \mathrm{~dB}$, in units of 5 , were added using the awgn function from Matlab's Communications System Toolbox.

\subsection{Brain cohort}

Brain magnetic resonance elastography data were prospectively acquired for a cohort of 48 healthy volunteer subjects (22 men and 26 women, ages 18-65), at seven frequencies, $30-60 \mathrm{~Hz}$ in steps of 5. The acquisition protocol is the same as that described for the healthy volunteers in Fehlner et al. (2016). All subjects gave their ethical consent as specified by the ethical review board.

For analysis we followed Dittmann et al. (2016) which applied a "sliding window" of three frequencies across a frequency band $(30,35$, and $40 \mathrm{~Hz}, 35,40$, and $45 \mathrm{~Hz}$, etc.). Here the sliding window was used to evaluate the stability of a three-frequency exam and investigate relationships in the data within and across frequencies.

\subsubsection{Stiffness and age measurements}

$\left|G^{*}\right|$ was reported for both whole brain and segmented white matter for each frequency set. Further, correlation to age, using Pearson's correlation coefficient $r$, was reported for each quantity. Some previous studies (e.g. Arani et al., 2015; Sack et al., 2009) have treated age in a more complex manner, building models and deriving both goodness of fit measurements and coefficient of determination $\mathbf{R}^{\mathbf{2}}$. In this initial study the focus is on evaluating two inversion algorithms, and model-building was deemed out-ofscope. The present $r$ is compared with previous $\mathbf{R}^{\mathbf{2}}$ in Discussion. Increase of $\left|G^{*}\right|$ values was expected across the frequency sets due to frequency-related dispersion. Again to keep the present study model-free, the slope across the sliding frequency windows was measured with a linear fit, and correlations of this quantity to age were also measured.

\subsubsection{Image quality measurements}

We incorporated three image quality measurements to investigate the quality of the present data set. In contrast to MRI magnitude images, empty space in phase images contains saltand-pepper rather than background noise, and this has led to the development of alternative signal-to-noise ratio measures for MRE. These alternative measures produce values as high as $\approx 750$ (Plewes et al., 2000) and as low as $\approx 3$ (McGarry et al., 2011). Among these measures some recent work has suggested that signal-to-noise ratio of derivative images, such as of the octahedral shear strain (OSS) (McGarry et al., 2011) or the Laplacian (Manduca et al., 2015), are better predictors of final image quality than signal-to-noise ratio of displacements.

SNR of such derivative images is of interest. However, widespread and robust methods of blind noise estimation exist that produce signal-to-noise ratio values which relate to mainstream, best-practice signal-to-noise ratio values, rather than relating only to their own sui generis scalings. There is no reason such methods cannot be applied to an image of MRE displacements or its derivatives. Here we use one of the most widely used noise estimation metrics in signal processing, that of Donoho and Johnstone (1995)

\section{$\hat{\sigma}=\operatorname{median}\left(\left|\psi_{J-1}\right|\right) / 0.6745$}

where $\psi_{J-1}$ is the finest band of wavelet coefficients in a $J$-level multi-resolution analysis. Not only is this a common measure but it would be expected to apply exceptionally well to magnetic resonance elastography as wavelet transforms are considered optimally sparse for wave images with discontinuities (Selesnick et al., 2005). This measure is applied to the anatomical regions using a mask. The power signal-to-noise ratio is then estimated as $S N R_{d B}=$ $20 \log _{10}\left(\sigma_{\text {signal }} / \sigma_{\text {noise }}\right)$. We estimated the signal-to-noise ratio of the displacement, OSS, and Laplacian images.

\section{Results}

\subsection{Simulation}

Fig. 1 shows simulation recovery at all five frequencies. Qualitatively, heterogeneous multifrequency direct inversion recovers target shapes more accurately than Multifrequency Dual Elasto-Visco Inversion. Fig. 2 shows results for the simulation study. Values are grouped by inversion method (Multifrequency Dual Elasto-Visco Inversion, heterogeneous multifrequency direct inversion) and data are plotted by target, noise level, and sliding frequency window. For both methods, the background material accurately reproduces the prescribed value of $\approx 3000 \mathrm{~Pa}$, as seen by the bottom line, until $\approx 20 \mathrm{~Hz}$. For the range of $50-20 \mathrm{~Hz}$, Multifrequency Dual ElastoVisco Inversion estimated $3154 \pm 32$ Pa while heterogeneous multifrequency direct inversion estimated $3067 \pm 11 \mathrm{~Pa}$.

Outside of target 2, the targets show variation with frequency on the order of 3\%, with an average standard deviation of $269 \mathrm{~Pa}$ for Multifrequency Dual Elasto-Visco Inversion and 174 Pa for heterogeneous multifrequency direct inversion respectively. Target 2 was an outlier in both cases with average standard deviation by frequency of $734 \mathrm{~Pa}$ and $383 \mathrm{~Pa}$ respectively. The targets also showed some sensitivity to noise: while estimates for heterogeneous multifrequency direct inversion large target at $50 \mathrm{~dB}$ noise averaged $9797 \mathrm{~Pa}$, this decreased slightly but stayed about $9000 \mathrm{~Pa}$ until $20 \mathrm{~dB}$. Multifrequency Dual Elasto-Visco Inversion showed more noise sensitivity, with an initial average of 10679 Pa but decreasing more sharply so that at $20 \mathrm{~dB}$ noise, only $8373 \mathrm{~Pa}$ was measured.

The smaller targets were not recovered accurately with either method, suggesting that below a threshold of $20 \mathrm{~mm}$ radius, accuracy is increasingly impeded by inaccurate readings at the boundary. In many cases, the targets were nonetheless distinguishable from background; in the case of heterogeneous multifrequency direct inversion, they were in fact all distinguishable, with means of $8-9 \mathrm{kPa}, 5-6 \mathrm{kPa}$, and 4-5 $\mathrm{kPa}$ respectively. In the Multifrequency Dual Elasto-Visco Inversion case, all targets except the first 


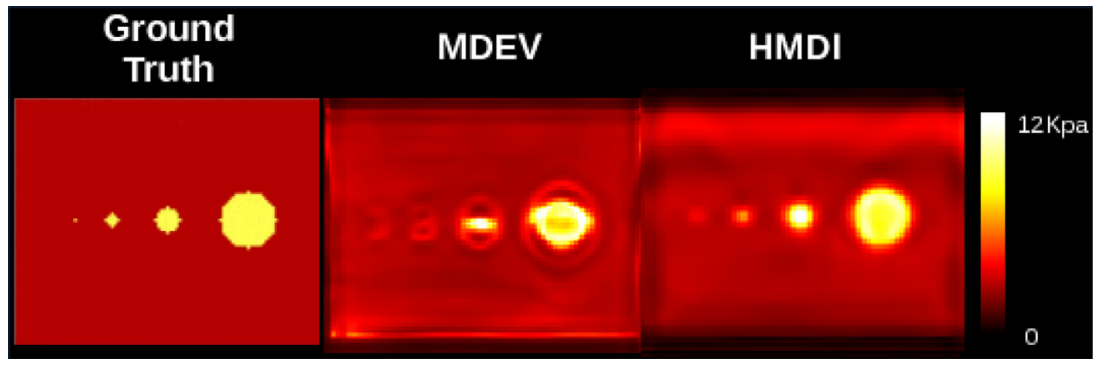

Fig. 1. Illustration of simulation denoising results at $30 \mathrm{~dB}$ noise: ground truth, MDEV method and HMDI method.

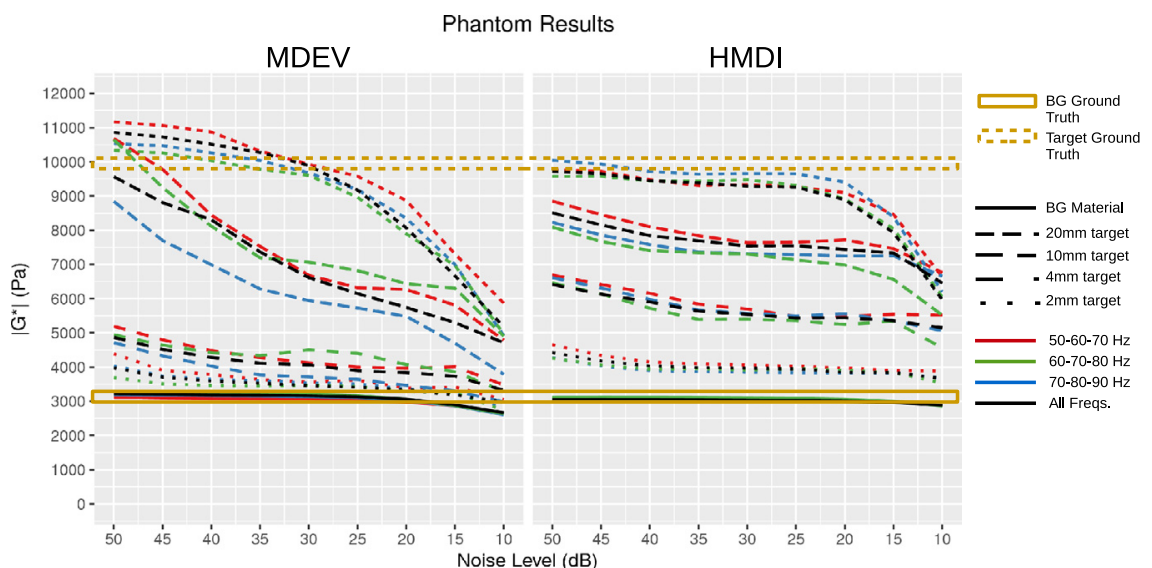

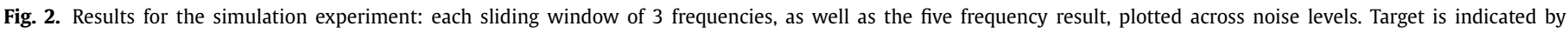
line pattern. Frequency is indicated by color.

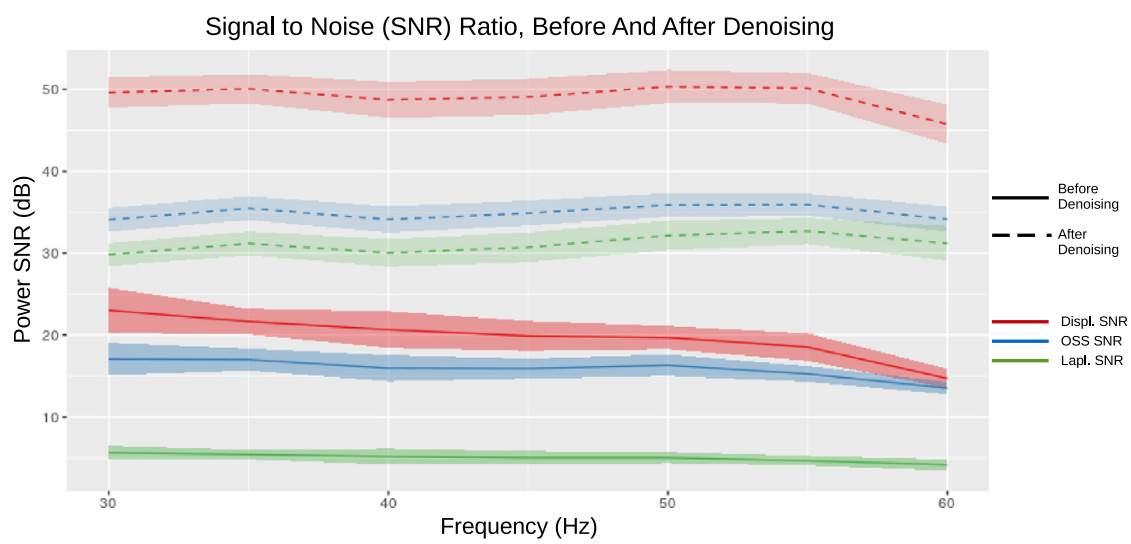

Fig. 3. Results for the brain SNR measurements. Solid lines indicate SNR measurements prior to denoising, and dashed lines indicate SNR measurements after denoising.

and second registered at $5 \mathrm{kPa}$ or lower. For all data, the average of all five frequencies sat in the center of the range of results.

\subsection{SNR results}

Fig. 3 show results for the signal-to-noise ratio measures. Displacement signal-to-noise ratio values, the equivalent of using a typical blind signal-to-noise ratio measure on Fouriertransformed magnetic resonance elastography output, showed results of $21.5 \pm 3.1 \mathrm{~dB}$. signal-to-noise ratio of the OSS image results were in the range of $15.6 \pm 1.4 \mathrm{~dB}$. signal-to-noise ratio of the Laplacian images were in the range of $4.6 \pm 0.6 \mathrm{~dB}$. After denoising, displacement signal-to-noise ratio measured $50.0 \pm 2.4 \mathrm{~dB}$, OSS image signal-to-noise ratio measured $34.2 \pm 1.0 \mathrm{~dB}$, and Laplacian image signal-to-noise ratio measured $29.8 \pm 1.7 \mathrm{~dB}$.

\subsection{Brain}

Fig. 4 shows images from the central slice of a subject chosen at random, by method and by frequency. The results at each frequency show the expected frequency dispersion. Qualitatively, the heterogeneous images show more stability but also more smoothness.

Results for the segmentation and co-registration of a second brain are shown in Fig. 5. Panel (a) shows the central slice of the acquisition in native space, in orthogonal views of $\mathrm{XY}, \mathrm{XZ}$ and $\mathrm{YZ}$. Panel (b) shows approximately the same region of the brain after co-registration to MNI space. Despite being only a $30 \mathrm{~mm}$ slab, the magnetic resonance elastography acquisition is accurately situated in MNI space. Panel (c) shows results for the SPM segmentation of this brain: the accompanying $\mathrm{T} 2$ image used for the segmentation is shown at left, followed by the segmentation results 


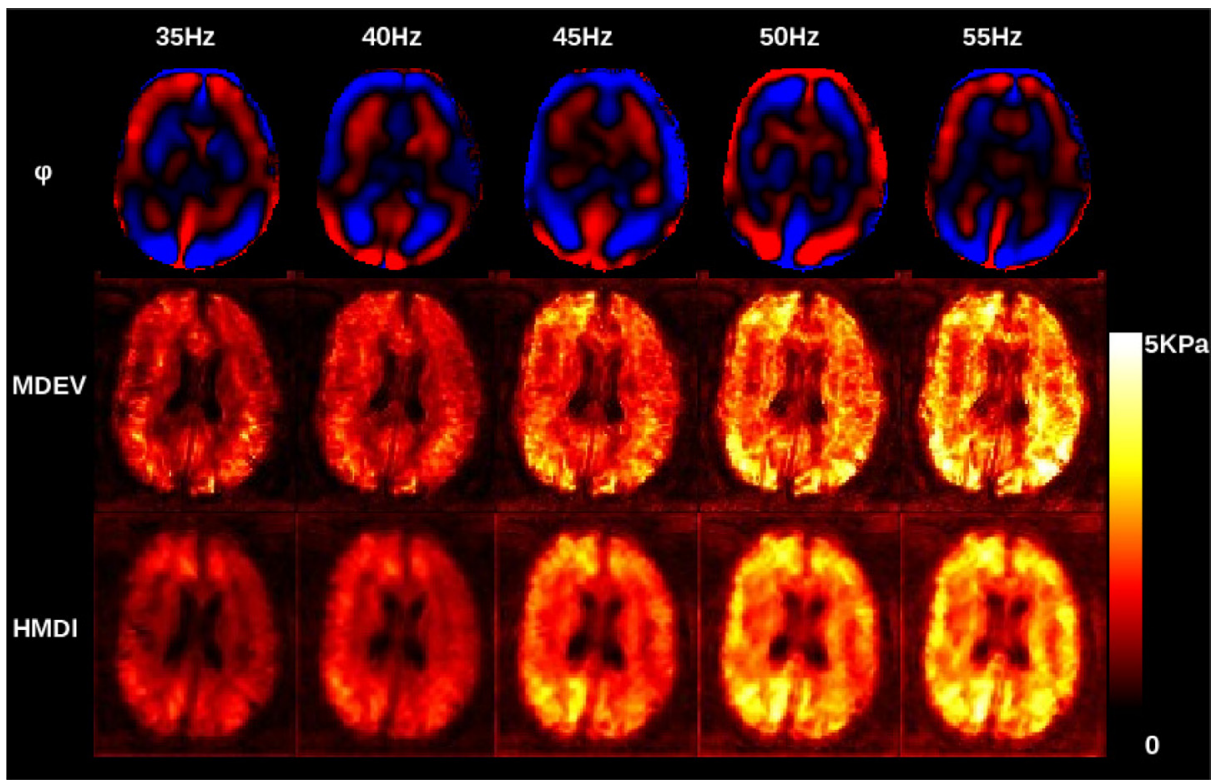

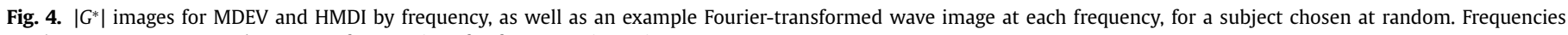
on the top row correspond to center frequencies of 3-frequency inversions.

(a) NATIVE SPACE

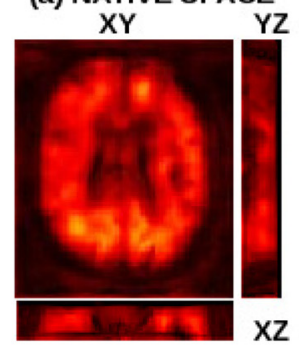

T2w Image

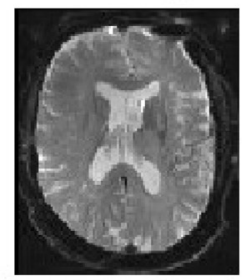

(b) MNI SPACE

YZ
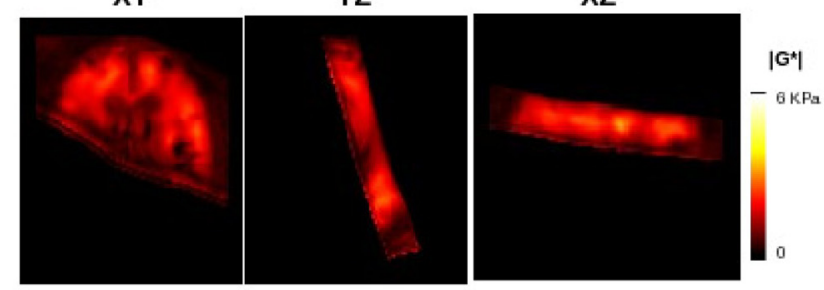

(c) T2 SEGMENTATION

GM

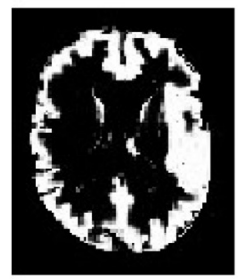

WM

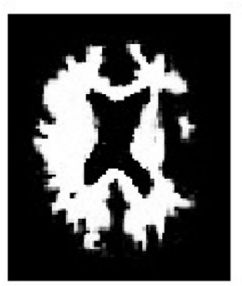

CSF

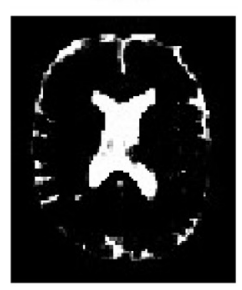

Fig. 5. Example images from segmentation of a second brain.

for gray matter (GM), white matter (WM), and cerebro-spinal fluid (CSF).

\subsubsection{Stiffness values and correlations to age}

Fig. 6 plots $\left|G^{*}\right|$ stiffness against sliding window frequency group, for each subject, for both whole brain and white matter segmentation. Inset within each plot is Pearson's $r$ results by frequency. For both methods, stiffness values rose by frequency in each set as predicted by known viscoelastic frequency dispersion. Negative correlation to age was more pronounced at higher frequencies. Both methods had comparable values at the lowest frequencies: for the $35 \mathrm{~Hz}$ inversions, Multifrequency Dual ElastoVisco Inversion $\left|G^{*}\right|$ was estimated at $1189 \pm 121 \mathrm{~Pa}$ and heterogeneous multifrequency direct inversion $\left|G^{*}\right|$ was $1022 \pm 136 \mathrm{~Pa}$. As the frequencies increased, heterogeneous multifrequency direct inversion stratified more, and increased more: for the $55 \mathrm{~Hz}$ inversions, Multifrequency Dual Elasto-Visco Inversion estimated $2376 \pm 193 \mathrm{~Pa}$ and heterogeneous multifrequency direct inversion estimated $2589 \pm 321 \mathrm{~Pa}$. Stratification was greater in the white matter analysis where Multifrequency Dual Elasto-Visco Inversion ranged from $1483 \pm 160$ Pa to $2670 \pm 230$ and heterogeneous multifrequency direct inversion ranged from $1305 \pm 161 \mathrm{~Pa}$ to $2979 \pm 312 \mathrm{~Pa}$.

To capture the relation between dispersion and age, Pearson's $r$ was calculated for the average slope across frequencies, by subject, for both whole brain and WM $\left|G^{*}\right|$. For whole brain, MDEV showed slope-to-age correlation of $r=0.38$; for heterogeneous multifrequency direct inversion $r=0.45$. In white matter MDEV showed slope-to-age correlation of $r=0.18$; for heterogeneous multifrequency direct inversion, $r=0.37$. 

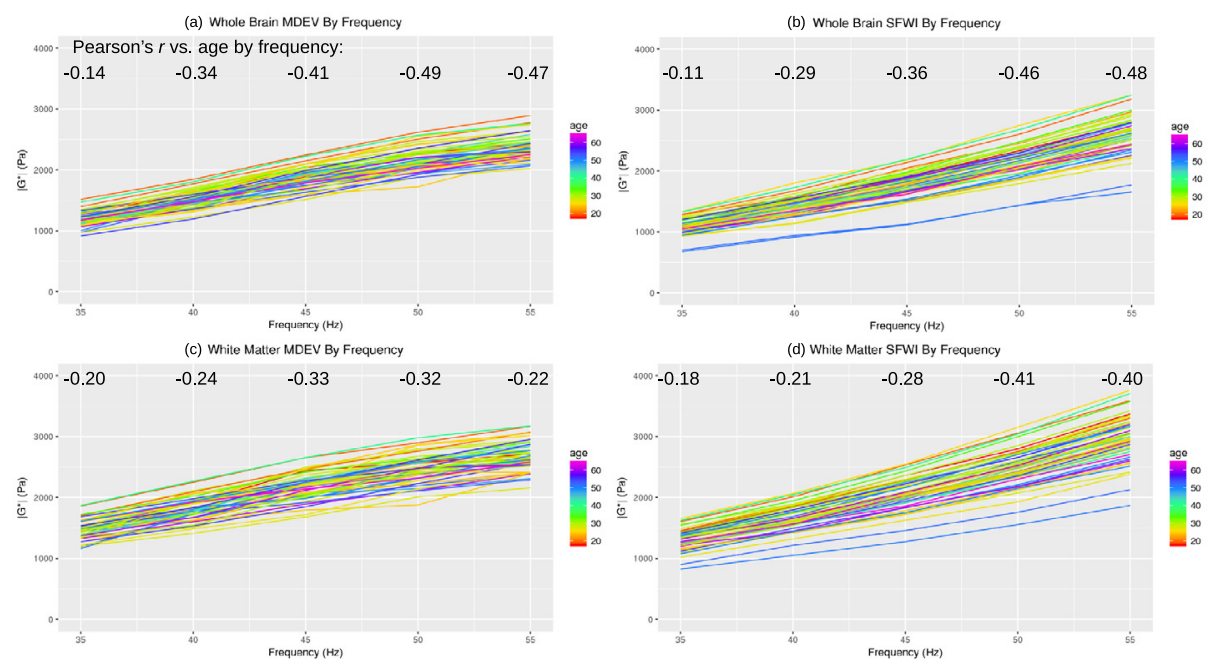

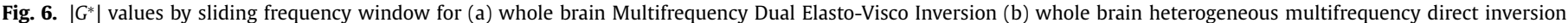
(c) white matter Multifrequency Dual Elasto-Visco Inversion (d) white matter heterogeneous multifrequency direct inversion.

\section{Discussion}

\subsection{Simulation experiment}

The simulation results as seen in Fig. 1 can be qualitatively compared with the Finite Element Method-based analyses found in Fig. 2 of Sánchez et al. (2010) and Fig. 6 of Barnhill et al. (2017), which both also contain either circular or cylindrical targets. Both studies like the present study show similar shape distortions using Helmholtz-type methods around the inserts. In Sánchez et al. (2010) and the present study, a gradient-inclusive direct inversion shows substantial improvement in the shape of recovered inclusions. We thus confirm the qualitative observations about the two methods in Sánchez et al. (2010).

The existence of features whose stiffness change is "detectable but not accurate" was first reported in Manduca et al. (2001), and is a continuing limitation in magnetic resonance elastography , where spatially extended maps always contain a trade-off between resolution and stability. Here the heterogeneous multifrequency direct inversion method estimated the large target with good accuracy and the $10 \mathrm{~mm}$ target with $\approx 90 \%$ accuracy, and remained consistent across noise levels in its estimations of the smaller targets, while the Multifrequency Dual Elasto-Visco Inversion method also preserved detectability for the most part, but showed greater sensitivity to noise.

The simulation experiment also shed some light into the relations between results by frequency: the noise removal process does introduce some variation by frequency, which the combination of greater numbers of frequencies helps to stabilize. This variation was on the order of $3 \%$, although the $10 \mathrm{~mm}$ target was an outlier, and had a range of $6-10 \%$. Despite this variation, this is an altogether positive finding for these methods, as it rules out the possibility that the strong frequency dependence in the brain results is a result of sensitivity to changes in noise values or wavelength.

\subsection{Image quality measures}

The use of mainstream signal-to-noise ratio measures shows immediate benefits in the interpretability of our signal-to-noise ratio results. Our wave images prior to denoising show signal-tonoise ratio in the $18-25 \mathrm{~dB}$ range, which is the same range of the $T 2 *$-weighted magnitude images of the same acquisition. The strain image shows lower signal-to-noise ratio, as is expected from taking a derivative, while the second-derivative Laplacian image is dominated by noise. The denoising procedure, however, produces consistent and steady improvements. Displacement signal-to-noise ratio is now around 50, considered very high, and the two derivative images are similar in signal-to-noise ratio and above 30 , which is also considered high. We interpret these results as showing our denoising procedures to be sufficient, not only for the images but for the derivatives used in the inversion.

\subsection{Brain experiment}

The brain results can be profitably interpreted in light of the simulation and signal-to-noise ratio findings. As regards comparison of methods, values are comparable in the lower end of the spectrum, but diverge for the highest frequency, with heterogeneous multifrequency direct inversion higher than Multifrequency Dual Elasto-Visco Inversion; this is explained by the dip in signalto-noise ratio values at the $60 \mathrm{~Hz}$ frequency, in combination with the greater noise-sensitivity of the Multifrequency Dual ElastoVisco Inversion method, and indeed inspection of the highest Multifrequency Dual Elasto-Visco Inversion group shows that the slope is slightly lower than at the other frequencies, suggesting that this one result was, in the end, impacted by signal-to-noise ratio concerns. The overall robustness of a multifrequency approach is supported by the internal consistency of the individual results in Fig. 6 , that is, subjects that were stiffer at one frequency also tended to be stiffer at another.

The heterogeneous multifrequency direct inversion group had two outliers, both older. One cause may have been challenges in the co-registration; systematic guidelines to verify successful coregistration will be produced in future work.

Most brain magnetic resonance elastography studies were performed at the relatively higher frequencies of 50 (Johnson et al., 2013) and 60 (Arani et al., 2015) Hz. Johnson et al. (2013) found a mean of $\approx 2.0 \mathrm{kPa}$ for gray matter $(\mathrm{GM})$ at and $\approx 2.7 \mathrm{kPa}$ for WM at $50 \mathrm{~Hz}$, and we find $\approx 2.1 \mathrm{kPa}$ and $2.5 \mathrm{kPa}$ respectively. Arani et al. (2015) found $\approx 2.6-2.8 \mathrm{kPa}$ for whole brain at $60 \mathrm{~Hz}$ and we find a slightly lower $\approx 2.4-2.6 \mathrm{kPa}$ at a slightly lower $55 \mathrm{~Hz}$. We report slightly lower values for a central frequency of $45 \mathrm{~Hz}$ than Dittmann et al. (2016) which found $2.18 \pm 0.2 \mathrm{kPa}$ to our $1.8-1.9 \mathrm{kPa}$ for $45 \mathrm{~Hz}$, however, both methods arrive at $\approx 2.2 \mathrm{kPa}$ for $50 \mathrm{~Hz}$. We conclude that our values are a good fit to the recent literature.

The multifrequency approach of a relatively large number of subjects yielded interesting insights into in vivo brain mechanics. 
While brain magnetic resonance elastography has been shown to be highly reproducible for a given individual, the literature reports a surprisingly wide variance in brain stiffness for healthy volunteers (Hiscox et al., 2016). Our study supports a wide range of individual variation. As seen in Fig. 6, the data have a wide range not from isolated outliers (though heterogeneous multifrequency direct inversion had two), but rather from the full range of the results being densely populated. One paradox of investigating interindividual variability, is that magnetic resonance elastography results can be made more similar through tight filtering bands, but at the expense of biasing the results. By using a noise-adaptive multi-scale filtering scheme, we preserve a large amount of bandwidth, enabling the full stiffness variation of the data to be observed with reduced bias.

The results for Pearson's $r$ of $\left|G^{*}\right|$ vs. age reach $\approx 0.4$ at high frequencies which is considered on the high end of moderate correlation. As we do not build a model, statistical inference from these correlations is not appropriate at present, beyond noting overall agreement with the findings in the models of Sack et al. (2009) and Arani et al. (2015). Particularly noteworthy from the present multifrequency study is the increasing correlation with frequency; again Fig. 6 is illustrative as to why. As the frequency increases, dispersion between young and old brains increases; Pearson's $r$ for slope-to-age also showed a similar correlation of $\approx 0.4$.

\subsection{Texture differences between MDEV and HMDI}

Finally, the present results should help refocus the debate in the magnetic resonance elastography community about the "local homogeneity assumption" along more productive lines. Many papers (e.g. Sinkus et al., 2010) suggest the homogeneity assumption is a cause of numerous limitations on image resolution, such more blurry and incoherent images. This reflects a fundamental misunderstanding of what the homogeneity assumption does. Neglecting the gradient in the solution means that where the solution does have non-neglectable gradient, it will be unstable and exaggerated; this will have a sharpening effect rather than a smoothing effect. The consequences of assuming local homogeneity are not lower resolution, but local instability, particular in regions of change in material properties, and we show in this paper that Multifrequency Dual Elasto-Visco Inversion shows less stability than heterogeneous multifrequency direct inversion when noise is combined with property change. Furthermore, we conclude that the numerical schemes used to implement the assumptions are at least as important as the assumptions themselves. In the present case, heterogeneous multifrequency direct inversion finds the least-squares solution with a global solve, which will produce the smoothest admissible solution. This produced a more stable, but also more smoothed, solution than an Helmholtz inversion which operates locally. A scheme that incorporates local heterogeneity but which evaluates locally is likely to resemble Multifrequency Dual ElastoVisco Inversion more than heterogeneous multifrequency direct inversion, and this will be shown in future work. In both cases, the summary statistics are very similar and either method could be used to progress future investigations in magnetic resonance elastography of the brain.

\subsection{Limitations}

We note two limitations of the study that were deemed outside the scope of this paper and reserved for future work. The first limitation concerns the recovery of modulus magnitude only. While heterogeneous multifrequency direct inversion magnitude can be validated against ground truth (and against Multifrequency
Dual Elasto-Visco Inversion in vivo), $\Im(G)$ does not currently provide meaningful information. Sánchez et al. (2010) note this as well and suggest constraining the minimization problem to force the imaginary component to be positive, effectively turning the inversion from an unconstrained to a constrained minimization problem, with corresponding increase in computational demands. However it is not clear that mere enforcement of non-negativity is sufficient constraint to produce a convincing loss modulus. Delivery of a clinically useful $\Im(G)$ is consequently reserved for a future investigation.

The second limitation involves divergence removal. The present study used a high-pass filter to remove low-frequency artefact rather than, for example, the divergence-free wavelet found in Barnhill et al. (2017). A high-pass approach is well established in magnetic resonance elastography, well understood, preserved the fine detail of the image and did not have a detrimental effect on simulation value recovery whose wavelengths are similar in range to magnetic resonance elastography acquisitions. A detailed comparison and validation of various divergence removal techniques in magnetic resonance elastography is underway and will be presented in future work.

\section{Conclusion}

Heterogeneous multifrequency direct inversion appears to be a valuable addition to the magnetic resonance elastography image reconstruction repertory. It applies a more sophisticated material model while maintaining the speed and convenience of a direct inversion. It produced highly similar results to Multifrequency Dual Elasto-Visco Inversion but showed more robustness to noise and to mechanical property change. The least-squares solve used for heterogeneous multifrequency direct inversion was stable but does create smoother images, and some desired magnetic resonance elastography applications require sharp boundaries. Future work will investigate sparsity promotion in the heterogeneous multifrequency direct inversion solve, as well as a reformulation in terms of local stencils. Our brain exam confirmed previous insights into aging and brain mechanical properties. By analyzing results from 48 subjects across 7 frequencies, we observed that agerelated differences grow with driving frequency due to viscosityrelated dispersion, demonstrated the robustness of the multifrequency paradigm, and added support to the case for wide-ranging inter-individual variation in stiffness values when measured with brain magnetic resonance elastography.

\section{Acknowledgements}

The authors are grateful to Lyam Hollis and the University of Edinburgh for the Finite Element Method simulations, and for correspondence with: Dr. Kristy Tan and Dr. Mark Wagshul of Albert Einstein School of Medicine; Dr. Mila Nikolova, CNRS; Dr. Monika Bahl, IIT Delhi; and Dr. Armando Manduca, Mayo Clinic. The authors are further grateful for support from: EU FORCE (Horizon 2020, PHC-11-2015), German Research Foundation (Sa901/17, Br 2235/8), and the Bundesministerium für Bildung und Forschung (BMBF 01GQ1408).

\section{Supplementary material}

Supplementary material associated with this article can be found, in the online version, at doi:10.1016/j.media.2018.03.003.

\section{References}

Arani, A., Murphy, M.C., Glaser, K.J., Manduca, A., Lake, D.S., Kruse, S.A., Jack, C.R. Ehman, R.L., Huston, J., 2015. Measuring the effects of aging and sex on regional brain stiffness with $\mathrm{mr}$ elastography in healthy older adults. Neuroimage 111, 59-64. 
Barnhill, E., Hollis, L., Sack, I., Braun, J., Hoskins, P.R., Pankaj, P., Brown, C., van Beek, E.J., Roberts, N., 2017. Nonlinear multiscale regularisation in $\mathrm{mr}$ elastography: towards fine feature mapping. Med. Image Anal. 35, 133-145.

Barnhill, E., Kennedy, P., Johnson, C.L., Mada, M., Roberts, N., 2014. Real-time 4d phase unwrapping applied to magnetic resonance elastography. Magn. Reson. Med. 73 (6). doi:10.1002/mrm.25332.

Braun, J., Guo, J., Lützkendorf, R., Stadler, J., Papazoglou, S., Hirsch, S., Sack, I., Bernarding, J., 2014. High-resolution mechanical imaging of the human brain by three-dimensional multifrequency magnetic resonance elastography at $7 \mathrm{t}$. Neuroimage 90, 308-314.

Dittmann, F., Hirsch, S., Tzschätzsch, H., Guo, J., Braun, J., Sack, I., 2016. In vivo wideband multifrequency $\mathrm{mr}$ elastography of the human brain and liver. Magn. Reson. Med. 76 (4), 1116-1126.

Donoho, D.L., Johnstone, I.M., 1995. Adapting to unknown smoothness via wavelet shrinkage. J. Am. Stat. Assoc. 90 (432), 1200-1224.

Eaton, J., Bateman, D., Hauberg, S., Wehbring, R., 2015. GNU octave version 4.0.0 manual: a high-level interactive language for numerical computations.

Evans, A.C., Janke, A.L., Collins, D.L., Baillet, S., 2012. Brain templates and atlases. Neuroimage 62 (2), 911-922.

Fehlner, A., Behrens, J.R., Streitberger, K.-J., Papazoglou, S., Braun, J., Bellmann-Strobl, J., Ruprecht, K., Paul, F., Würfel, J., Sack, I., 2016. Higher-resolution mr elastography reveals early mechanical signatures of neuroinflammation in patients with clinically isolated syndrome. J. Magn. Reson. Imaging 44 (1), 51-58.

Fehlner, A., Hirsch, S., Weygandt, M., Christophel, T., Barnhill, E., Kadobianskyi, M., Braun, J., Bernarding, J., Lützkendorf, R., Sack, I., et al., 2017. Increasing the spatial resolution and sensitivity of magnetic resonance elastography by correcting for subject motion and susceptibility-induced image distortions. J. Magn. Reson. Imaging 46 (1), 134-141.

Friston, K., Ashburner, J., Frith, C.D., Poline, J.-B., Heather, J.D., Frackowiak, R.S., et al., 1995. Spatial registration and normalization of images. Hum. Brain Mapp. 3 (3), 165-189.

Gauss, C.F., 1816. Bestimmung der genauigkeit der beobachtungen. Astronomi 1, 185-197.

Gudbjartsson, H., Patz, S., 1995. The rician distribution of noisy mri data. Magn. Reson. Med. 34 (6), 910-914.

Guo, J., Hirsch, S., Fehlner, A., Papazoglou, S., Scheel, M., Braun, J., Sack, I., 2013. Towards an elastographic atlas of brain anatomy. PloS one 8 (8), e71807.

Hirsch, S., Braun, J., Sack, I., 2016. Magnetic Resonance Elastography: Physical Background and Medical Applications. John Wiley \& Sons.

Hiscox, L.V., Johnson, C.L., Barnhill, E., McGarry, M.D., Huston 3rd, J., van Beek, E.J., Starr, J.M., Roberts, N., 2016. Magnetic resonance elastography (mre) of the human brain: technique, findings and clinical applications. Phys. Med. Biol. 61 (24), R401.

Hollis, L., Barnhill, E., Conlisk, N., Thomas-Seale, L.E., Roberts, N., Pankaj, P., Hoskins, P.R., 2016. Finite element analysis to compare the accuracy of the direct and mdev inversion algorithms in mr elastography.. IAENG Int. J. Comput. Sci. 43 (2).

Honarvar, M., Sahebjavaher, R., Sinkus, R., Rohling, R., Salcudean, S.E., 2013. Curl-based finite element reconstruction of the shear modulus without assuming local homogeneity: time harmonic case. IEEE Trans. Med. Imaging 32 (12), 2189-2199.

Johnson, C.L., McGarry, M.D., Gharibans, A.A., Weaver, J.B., Paulsen, K.D., Wang, H. Olivero, W.C., Sutton, B.P., Georgiadis, J.G., 2013. Local mechanical properties of white matter structures in the human brain. Neurolmage 79, 145-152.
Knowles, I., Renka, R.J., 2014. Methods for numerical differentiation of noisy data. Electron. J. Differ. Eq. 21 (2012), 235-246.

Manduca, A, Lake, D. S, Huynh, KT, Eon, R.S., Annoni, E.M., Ehman, R.L., 2015. Consistent snr measures for magnetic resonance elastography. In: Proceedings of the 23rd Annual Meeting of the International Society for Magnetic Resonance in Medicine (ISMRM). ISMRM.

Manduca, A., Oliphant, T., Dresner, M., Mahowald, J., Kruse, S., Amromin, E., Felmlee, J., Greenleaf, J., Ehman, R., 2001. Magnetic resonance elastography: non-invasive mapping of tissue elasticity. Med. Image Anal. 5 (4), 237-254.

Mariappan, Y.K., Glaser, K.J., Ehman, R.L., 2010. Magnetic resonance elastography: a review. Clin. Anatomy 23, 497-511.

McGarry, M., Van Houten, E., Perrinez, P., Pattison, A., Weaver, J., Paulsen, K., 2011. An octahedral shear strain-based measure of snr for 3d mr elastography. Phys. Med. Biol. 56 (13), N153.

McGarry, M.D., 2013. Improvement and evaluation of nonlinear inversion MR elastography. Dartmouth College.

Oliphant, T.E., Manduca, A., Ehman, R.L., Greenleaf, J.F., 2001. Complex-valued stiffness reconstruction for magnetic resonance elastography by algebraic inversion of the differential equation. Magn. Reson. Med. 45 (2), 299-310.

Papazoglou, S., Hamhaber, U., Braun, J., Sack, I., 2008. Algebraic helmholtz inversion in planar magnetic resonance elastography. Phys. Med. Biol. 53 (12), 3147.

Papazoglou, S., Hirsch, S., Braun, J., Sack, I., 2012. Multifrequency inversion in magnetic resonance elastography. Phys. Med. Biol. 57 (8), 2329.

Park, E., Maniatty, A.M., 2006. Shear modulus reconstruction in dynamic elastography: time harmonic case. Phys. Med. Biol. 51 (15), 3697.

Plewes, D.B., Bishop, J., Samani, A., Sciarretta, J., 2000. Visualization and quantification of breast cancer biomechanical properties with magnetic resonance elastography. Phys. Med. Biol. 45 (6), 1591.

Sack, I., Beierbach, B., Wuerfel, J., Klatt, D., Hamhaber, U., Papazoglou, S., Martus, P. Braun, J., 2009. The impact of aging and gender on brain viscoelasticity. Neuroimage 46 (3), 652-657.

Sack, I., Jöhrens, K., Würfel, J., Braun, J., 2013. Structure-sensitive elastography: on the viscoelastic powerlaw behavior of in vivo human tissue in health and disease. Soft Matter 9 (24), 5672-5680.

Sánchez, C., Drapaca, C., Sivaloganathan, S., Vrscay, E., 2010. Elastography of biological tissue: direct inversion methods that allow for local shear modulus variations. Image Anal. Recognit. 195-206.

Selesnick, I.W., Baraniuk, R.G., Kingsbury, N.C., 2005. The dual-tree complex wavelet transform. Signal Process. Mag., IEEE 22 (6), 123-151.

Sinkus, R., Daire, J.-L., Van Beers, B.E., Vilgrain, V., 2010. Elasticity reconstruction: beyond the assumption of local homogeneity. Comptes Rendus Mécanique 338 (7-8), 474-479.

Sinkus, R., Tanter, M., Catheline, S., Lorenzen, J., Kuhl, C., Sondermann, E., Fink, M., 2005. Imaging anisotropic and viscous properties of breast tissue by magnetic resonance-elastography. Magn. Reson. Med. 53 (2), 372-387.

Streitberger, K.-J., Reiss-Zimmermann, M., Freimann, F.B., Bayerl, S., Guo, J., Arlt, F., Wuerfel, J., Braun, J., Hoffmann, K.-T., Sack, I., 2014. High-resolution mechanical imaging of glioblastoma by multifrequency magnetic resonance elastography. PloS one 9 (10), e110588.

Szabo, T.L., 1995. Causal theories and data for acoustic attenuation obeying a frequency power law. J. Acoust. Soc. Am. 97, 14.

Van Houten, E., Paulsen, K., Miga, M., Kennedy, F., Weaver, J., et al., 1999. An overlapping subzone technique for mr-based elastic property reconstruction. Magn. Reson. Med. 42 (4), 779-786. 